\title{
EDUCAÇÃO FINANCEIRA A SERVIÇO DA CIDADANIA
}

\author{
FINANCIAL EDUCATION FOR CITIZENSHIP
}

\author{
João Paulo Attie ${ }^{1}$ \\ Nilson Setsuo Ozawa ${ }^{2}$ \\ Nadir Santos Freitas ${ }^{3}$
}

\begin{abstract}
RESUMO: Neste artigo, apresentamos os resultados de uma pesquisa realizada com estudantes do ensino médio, que teve como objetivo identificar o conhecimento dos mesmos a respeito da Educação Financeira. $O$ trabalho é fruto da participação de um dos autores no Mestrado Profissional em Matemática em Rede Nacional (PROFMAT), e teve como base teórica autores como Kistemann (2011), Skovsmose (2000), Barbosa (2004) e Bassanezi (2012), além de textos institucionais, tais como o relatório da Organização para a Cooperação e Desenvolvimento Econômico (OECD, 2005), os Parâmetros Curriculares Nacionais, os PCN (BRASIL, 1997; 1999) e a Proposta Curricular estadual (SÃO PAULO, 2008). A pesquisa de cunho qualitativo, do tipo descritiva, de acordo com Gil (2002), teve como instrumento de coleta de dados um questionário, cujas perguntas foram elaboradas com base na contextualização e na modelagem. $O$ instrumento foi aplicado a um grupo de sessenta e dois estudantes do segundo ano do Ensino Médio, de uma escola pública localizada no interior do Estado de São Paulo e os dados foram observados a partir da análise de conteúdo (BARDIN, 1997). Como resultados, podemos apontar a compreensão dos participantes a respeito de conteúdos básicos como juros e operações, mas um desconhecimento temerário em relação aos tipos de financiamento e ao comportamento da função exponencial, nos levando à conclusão da necessidade de maior presença da contextualização, da modelagem e da educação financeira no processo de ensino.
\end{abstract}

PALAVRAS-CHAVE: Educação Financeira. Modelagem Matemática. Ensino de Matemática.

\begin{abstract}
In this article, we present the results of a survey carried out with high school students, which aimed to identify their knowledge about Financial Education. The work results from the participation of one of the authors in the Professional Master's Degree in Mathematics in a National Network (Programa de Mestrado Profissional em Matemática em Rede Nacional, PROFMAT), and was theoretically based on authors such as Kistemann (2011), Skovsmose (2000), Barbosa (2004) and Bassanezi (2012), as well as institutional texts, such as the Organization for Economic Cooperation and Development report's (OECD, 2005), the National Curriculum Parameters, called PCN (BRASIL, 1997; 1999) and a District Curriculum Proposal (SÃO PAULO, 2008). The research, of qualitative nature, a descriptive type, according to Gil (2002), had as a data collection instrument a questionnaire, whose questions were elaborated based on contextualization and on modeling. The instrument was applied to a group of sixty-two second-year high school students from a public school located in the interior of the state of São Paulo, Brazil and the data were observed from the content analysis (BARDIN, 1997). As a result, we can point out the participants' understanding of basic contents such as interest and operations, but a reckless lack of knowledge regarding the types of financing and the behavior of the exponential function, leading us to the conclusion of the need for greater presence of contextualization, of modeling and financial education in the teaching process.
\end{abstract}

KEYWORDS: Financial Education. Mathematical Modeling. Mathematics Teaching.

\footnotetext{
${ }^{1}$ Universidade Federal de Sergipe. E-mail: jpattie@mat.ufs.br

(iD) https://orcid.org/0000-0001-8411-4168

${ }^{2}$ Rede Estadual de São Paulo. E-mail: niljap@yahoo.com.br

(iD) https://orcid.org/0000-0002-8343-1775

${ }^{3}$ Rede Estadual de Alagoas. E-mail: nadir.matematica@hotmail.com

(iD) https://orcid.org/0000-0001-9505-3356

- Informações completas da obra no final do artigo
} 


\section{Introdução}

Vivemos em um país com uma das maiores taxas de má distribuição de renda e desigualdade do mundo. Segundo o relatório do PNUD, o Programa das Nações Unidas para o Desenvolvimento Humano, publicado em 2019, o Brasil, entre 189 países, é o $2^{\circ}$ país do mundo com maior concentração de renda do planeta, perdendo apenas para o Catar (ONU, 2019).

Consideramos que a superação desse quadro não se dará apenas pelo viés econômico, e depende essencialmente de uma educação voltada para a cidadania. Simultaneamente a esse contexto, o planeta atravessa o real perigo do esgotamento ambiental, devido à velocidade e intensidade do consumo (ONU, 2020). Dessa forma, concordamos que "a sociedade do século XXI não pode prescindir de discutir uma educação financeira, bem como significados em torno de ideias, que se embasam em práticas conscientes de consumo" (KISTEMANN, 2011, p. 30). Além disso, consideramos que alguns outros aspectos são fundamentais, como o planejamento financeiro, a tomada de decisões acerca das ações praticadas pelo indivíduo, enquanto cidadão e enquanto consumidor, quando almeja adquirir um produto no qual deverá ter conhecimento para não ser ludibriado, bem como, desenvolver hábitos que propiciem a arte de manejar criticamente os objetos matemáticos de cunho financeiro-econômicos.

Nesse contexto, o objetivo deste artigo é apresentar os resultados de uma pesquisa que foi realizada como parte no desenvolvimento da dissertação de um dos autores no Mestrado Profissional em Matemática em Rede Nacional (PROFMAT), e que teve como finalidade identificar o conhecimento de estudantes a respeito da educação financeira. $O$ trabalho foi desenvolvido com um grupo de 62 estudantes do $2^{\circ}$ ano do Ensino Médio de uma escola pública da rede estadual de São Paulo, abordando os conceitos de matemática financeira que estivessem atrelados a alguns aspectos pertinentes para a construção da educação financeira.

Entre os conceitos principais que fundamentaram o trabalho, aparecem, além das noções principais em relação à Educação Financeira, também a necessidade da contextualização no ensino de matemática, com a consequente demanda da utilização da modelagem, em autores como Kistemann (2011), Skovsmose (2000), Barbosa (2004) e 
Bassanezi (2012), entre outros. Além dessas fontes, procuramos elementos em alguns textos institucionais, tais como o relatório da Organização para a Cooperação e Desenvolvimento Econômico (OECD, 2005), os Parâmetros Curriculares Nacionais, os PCN (BRASIL, 1997; 1999) e a Proposta curricular estadual (SÃO PAULO, 2008).

À primeira vista, podemos apontar alguns resultados importantes, tais como a compreensão do conceito básico de juros, aliado ao desconhecimento do tipo de juro aplicado em cada caso, bem como da relação existente entre a taxa de juros e algum tipo de função. O processo de tomada de decisão dos estudantes, ao longo do trabalho, foi sendo refinado e contou com o acréscimo de alguns elementos tais como a análise e a comparação de benefícios e de prejuízos em cada decisão.

\title{
O PROFMAT
}

O Mestrado Profissional em Matemática em Rede Nacional (PROFMAT), foi o primeiro curso de pós-graduação stricto sensu, no Brasil, oferecido no formato semipresencial. Além disso, ainda se apresenta como o primeiro curso de mestrado profissional oferecido em rede nacional.

O programa, criado em 2011, surgiu a partir de uma demanda legal, a partir das políticas públicas brasileira, já que o PROFMAT

\begin{abstract}
Vem ao encontro do Plano Nacional de Educação (PNE), Lei № 13.005, de 25 junho de 2014, que coloca em sua Meta 16: formar, em nível de pós-graduação, 50\% (cinquenta por cento) dos professores da Educação Básica, até o último ano de vigência deste PNE e garantir a todos (as) os (as) profissionais da Educação Básica formação continuada em sua área de atuação, considerando as necessidades, demandas e contextualizações dos sistemas de ensino. Além disso, o PROFMAT também atende as metas 14, 17 e 18 que tratam, respectivamente, de elevar o número de matrículas na pós-graduação stricto sensu, da valorização do professor e do plano de carreira (SBM, 2017, p. 04).
\end{abstract}

Fazem parte do programa uma gama de Instituições de Ensino Superior, vinculadas entre si pela Universidade Aberta do Brasil (UAB) e pela Coordenação de Aperfeiçoamento Pessoal de Nível Superior (CAPES). A coordenação do PROFMAT é exercida pela Sociedade Brasileira de Matemática (SBM), contando com o apoio do Instituto Nacional de Matemática Pura e Aplicada (IMPA).

O público-alvo específico do programa engloba o conjunto dos professores de Matemática em exercício na Educação Básica, especialmente de escolas públicas, que pretendem aprimorar sua formação profissional, e o destaque maior é dado ao domínio do 
conteúdo matemático. Dessa forma, o programa tem como objetivo "proporcionar formação matemática aprofundada e relevante para a docência na Educação Básica, visando dar ao egresso qualificação certificada para o exercício da profissão de professor de Matemática" (SBM, 2017, p. 03).

Segundo dados da SBM, de 2017, o Programa foi recomendado pela CAPES, reconhecido pelo Conselho Nacional de Educação (CNE) e validado pelo Ministério da Educação (MEC) com nota 5 (nota máxima para programas de mestrado), tendo, até aquele ano, ofertado mais de 10.000 vagas e apresentando uma quantidade de cerca de 3.000 alunos formados.

\section{Educação financeira, contextualização e modelagem}

Consideramos importante ressaltar que as expressões "Educação Financeira" e "Matemática Financeira" não devem ser tratadas como sinônimos, pois, enquanto a primeira expressão deve se pautar pela "compreensão, capacidade, comportamentos, atitudes e valores que permitam aos alunos tomar decisões financeiras seguras e efetivas" (MAUÉS, 2011, p. 03), a segunda pode ser descrita como um dos ramos da Matemática Aplicada que estuda o comportamento das finanças, procurando modelar e quantificar transações que envolvam ou resultem em valor monetário. A despeito de serem conceitos diferentes, é evidente que, em nosso trabalho, ponderamos que "a compreensão de elementos da Matemática Financeira é essencial para que se tenha uma boa Educação Financeira" (OZAWA, 2013, p. 48).

Nesse panorama, poderíamos apontar que a importância da Educação Financeira no processo de ensino de matemática surge na intersecção entre a necessidade de uma tomada de decisão e nos conteúdos pertencentes à matemática financeira.

Nesse contexto, consideramos a necessidade de que a Educação Financeira faça parte da formação de um cidadão crítico, autônomo e consciente de seus deveres e direitos, pois

a Educação Financeira não consiste somente em aprender a economizar, cortar gastos, poupar e acumular dinheiro, é muito mais que isso. É buscar uma melhor qualidade de vida tanto hoje quanto no futuro, proporcionando a segurança material necessária para obter uma garantia para eventuais imprevistos (TEIXEIRA, 2015, p. 13).

Segundo a Organização para a Cooperação e Desenvolvimento Econômico: 
A educação financeira é o processo pelo qual consumidores e investidores melhoram sua compreensão sobre conceitos e produtos financeiros e, por meio de informação, instrução e orientação objetiva, desenvolvem habilidades e adquirem confiança para se tornarem mais conscientes das oportunidades e dos riscos financeiros, para fazerem escolhas bem-informadas e saberem onde procurar ajuda ao adotarem outras ações efetivas que melhorem o seu bem-estar e a sua proteção (OECD, 2009, p. 02).

Nossa defesa, portanto, é da inserção da Educação Financeira no processo de ensino, em uma abordagem que não se limite ao estímulo de "poupar para consumir" (OLIVEIRA, 2017, p. 112), mas com o objetivo de proporcionar reflexões aos alunos, de forma que o seu processo de tomada de decisão seja feito de forma consciente. Neste panorama, o presente trabalho buscou oferecer aos participantes uma situação que pudesse fazer parte de seu contexto cotidiano, na qual fosse exigida uma decisão dos mesmos. A partir das respostas obtidas, pudemos reelaborar a situação em sala de aula, buscando um modelo matemático que pudesse responder às eventuais dúvidas-

Em relação à contextualização, seguimos o conceito apresentado por Skovsmose (2000), segundo o qual existem três tipos de contextos possíveis para as atividades escolares: a Matemática pura, quando a situação pertence integralmente à matemática acadêmica; o contexto da semi-realidade, que ocorre quando a situação envolve elementos do cotidiano ou do mundo físico, mas tratando de situações fictícias e, por fim, a realidade, que acontece quando se descrevem situações que efetivamente ocorrem na vida diária e/ou científica. Assim, existem determinadas situações, no cotidiano do aluno, em que é possível utilizar o conhecimento da Matemática, e esses cálculos matemáticos, quando assim utilizados, na maioria dos casos servem para validar de forma numérica certas situações. Em nosso caso, nos aproveitamos de uma dessas situações reais que precisam de cálculos matemáticos e que, portanto, envolvem de maneira implícita ou explícita algum tipo de conceito matemático.

Ainda que vários autores defendam a utilização da contextualização no ensino de matemática, argumentando pela necessidade daquela ensinada no ambiente escolar incluir situações com referências na realidade, tais como D'Ambrosio (2001), Bassanezi (2012), e Machado (1990), além dos próprios documentos oficias: Parâmetros Curriculares Nacionais (PCN) e Base Nacional Comum Curricular (BNCC), consideramos o fato de que a disciplina talvez seja a única que pode prescindir da utilização dos fenômenos do mundo físico, pois:

Por mais que o desenvolvimento e a utilidade da matemática estejam fortemente vinculados às demandas da realidade, ela é a única disciplina em que o professor 
pode, caso assim o deseje (e essa opção pode ou não ser consciente e intencional), explanar seus conteúdos sem qualquer conexão com o mundo sensível. Nas demais disciplinas, o aluno possui algum entendimento precedente acerca dos assuntos abordados. Na matemática escolar ... esse entendimento não é compulsório ... a disciplina ainda se conserva como sendo a única em que aparece a possibilidade de não vinculação com o mundo (ATTIE, 2013, p. 24-25).

A utilização de contextos reais no ensino demanda a busca de modelos matemáticos que possam explicar quantitativamente aquela situação. Desta forma, concordamos que

A modelagem matemática é simplesmente uma estratégia utilizada para obtermos
alguma compreensão acerca de determinadas situações reais e assim, no processo
de reflexão sobre a porção da realidade, devemos selecionar apenas as variáveis
consideradas essenciais para, depois disso, procurarmos uma formalização que
explique as relações que envolvem essas variáveis (BASSANEZI, 2012, p. 10-11).

Considerando que um modelo matemático é um conjunto consistente de equações ou estruturas matemáticas, elaborado para corresponder a algum fenômeno, que pode ser físico, biológico, social, psicológico ou conceitual, a situação descrita aos alunos na pesquisa envolve a compra de um carro, e o modelo elaborado após aplicação dos questionários buscou apresentar procedimentos possíveis, utilizando equações simples e conceitos básicos de Matemática Financeira.

\section{Documentos públicos: PCN e BNCC}

A partir da implementação das Leis de Diretrizes e Bases da Educação, em 1996, foram introduzidos, em 1998, os Parâmetros Curriculares Nacionais, conhecidos como PCN, com o propósito de orientar a introdução de um currículo mínimo comum a todas as escolas. De acordo com o Ministério da Educação, os PCN são

Um documento introdutório que se propõe a apresentar as linhas norteadoras que constituem uma proposta de reorientação curricular oferecida pelo MEC às secretarias de educação, às escolas, às instituições de formação de professores, aos institutos de pesquisa, editoras e a todas as pessoas interessadas em educação no Brasil (BRASIL, 1998, p. 09).

No caso particular da Matemática, os parâmetros específicos da área (BRASIL, 1997) apresentam uma forte defesa de um ensino em que a Matemática fosse, sempre que possível, contextualizada, incentivando a utilização de atividades em que o conhecimento matemático pudesse ser utilizado de forma a ajudar a resolver problemas do cotidiano.

Os Parâmetros Curriculares Nacionais do Ensino Médio (PCNEM - BRASIL, 1999) apresentam competências e habilidades que foram escolhidas para o desenvolvimento 
deste trabalho, a partir de propostas como ler, interpretar e utilizar representações matemáticas (tabelas, gráficos, expressões e outras), identificar o problema (compreender enunciados, formular questões, entre outras dificuldades), procurar, selecionar e interpretar informações relativas ao problema, interpretar e criticar resultados numa situação concreta e aplicar conhecimentos e métodos matemáticos em situações reais, entre outras (BRASIL, 1999).

Em relação à Educação Financeira, especificamente, o Decreto-lei executivo 7397 (BRASIL, 2010), sancionado em 22 de dezembro de 2010, passa a considerar esse conteúdo como parte do currículo escolar, tendo como objetivo orientar o aluno a controlar melhor suas finanças no futuro, melhorando seu padrão de qualidade de vida. A necessidade do ensino de Educação Financeira é também atestada por Negri (2010), que mostra essa importância em conformidade tanto com os PCN, quanto com a Lei de Diretrizes e Bases da Educação Nacional, a LDB (BRASIL, 1996).

Em relação a outros documentos públicos, consideramos necessário esclarecer que, como a pesquisa foi realizada antes da publicação da BNCC (BRASIL, 2017), esse texto não faz parte da fundamentação teórica. Mesmo assim, incluímos especificamente neste artigo alguns aspectos do documento, pois a BNCC estabelece a Educação Financeira e a Educação para o Consumo como habilidades obrigatórias entre os componentes curriculares. Assim, neste documento normativo, entre as habilidades e competências esperadas, aparece a necessidade de "resolver e elaborar problemas, envolvendo cálculo de porcentagens, incluindo o uso de tecnologias digitais" (BRASIL, 2017. p. 311).

\section{Metodologia}

O trabalho foi desenvolvido em três etapas, sendo que o primeiro passo abrangeu a realização das pesquisas bibliográficas para a fundamentação teórica. O segundo passo, foi a realização de uma pesquisa de caráter qualitativo, por meio de um estudo de campo, onde aplicamos um questionário a um grupo de 62 estudantes do $2^{\circ}$ ano do Ensino Médio de uma escola pública da rede estadual de São Paulo. A partir desse panorama, a pesquisa pode ser apontada como sendo do tipo descritiva, pois tivemos como "objetivo primordial a descrição das características de determinada população" (GIL, 2002, p. 42).

Pela natureza do fenômeno estudado e pelas particularidades da abordagem realizada, optamos por uma análise qualitativa de dados, a partir do conteúdo, de acordo 
com Bardin (1977). A análise de conteúdo é, segundo a autora "um conjunto de técnicas de análise das comunicações visando a obter, por procedimentos sistemáticos e objetivos de descrição do conteúdo das mensagens, indicadores (quantitativos ou não) que permitam a inferência de conhecimentos relativos às condições de produção/recepção (variáveis inferidas) destas mensagens" (BARDIN, 1977, p. 42).

Antes da elaboração do questionário, foram colhidas sugestões dos alunos em relação ao assunto, tendo sido escolhido, a partir daí, o tema "compra de um automóvel". Procuramos seguir, no modelo matemático desenvolvido e apresentado aos estudantes no questionário, as orientações da Proposta Curricular do Estado de São Paulo para o Ciclo II e para o Ensino Médio, que abordam temas como as sequências numéricas e progressão aritmética (SÃO PAULO, 2008).

Por fim, o terceiro passo consistiu na análise dos dados obtidos na coleta, gerando a produção textual que buscou confrontar a teoria estudada com os dados obtidos. $O$ questionário compreendia apenas duas questões, uma relacionada às possibilidades para a compra de um automóvel e outra solicitando a justificativa para a alternativa escolhida.

Um dado importante a considerar é o de que os alunos não haviam tido contato em nenhum momento, dentro da disciplina curricular de Matemática, o tópico Matemática Financeira, sugerido no primeiro ano do ensino médio. Outro fato observado durante a pesquisa é que a maioria dos alunos utilizavam calculadora, subtraindo o valor do montante pelo capital, mostrando domínio no conhecimento deste material.

Em relação à faixa etária, a maioria dos alunos, 32 deles $(51,61 \%)$ tinha 16 anos de idade na época da aplicação do questionário, enquanto 22 estudantes (35,48\%) tinham 15 anos de idade e 8 alunos (12,91\%) tinham 17 anos de idade. A pesquisa foi realizada no ano de 2012, em uma escola no centro da cidade de Mogi das Cruzes, e, em relação à participação dos estudantes, foram apresentados Termos de Consentimento Livre e Esclarecido, que retornaram com a anuência dos responsáveis. Os participantes foram aqueles que mostraram interesse em se aprofundar e ter conhecimento da Matemática Financeira, com o objetivo declarado de planejar e comprar um carro.

\section{Resultados}

Em relação ao questionário em si, foram apresentadas algumas opções em relação à compra de um automóvel e, foi solicitado aos estudantes que escolhessem uma 
alternativa e justificassem sua opção. A situação apresentada era a de que o aluno dispusesse de $R \$ 10.000,00$, e diante da oportunidade da compra de um carro no valor de $R \$ 30.000,00$, qual opção de compra escolheria, entre as seguintes:

a) $R \$ 10.000,00$ de entrada e saldo em 36 meses com valor da prestação de $R \$$ 761,21 ;

b) $R \$ 10.000,00$ de entrada e saldo em 48 meses com valor de prestação de $R \$$ 691,32 ;

c) $R \$ 10.000,00$ de entrada e saldo em 60 meses com valor de prestação de $R \$$ 532,79 ;

d) Sem entrada, saldo em 60 meses, sem entrada com valor de prestação de $\mathrm{R} \$$ 795,50;

e) Esperar e comprar à vista (OZAWA, 2013, p. 37).

Entre as respostas obtidas, houve um inequívoco predomínio pela opção "e": esperar e comprar à vista, em um total de 42 respostas $(67,74 \%$ do total), conforme podemos observar na Tabela 1. Entre as justificativas encontradas, a mais frequente foi a de que teriam um maior desconto em caso de pagamento à vista, desconsiderando, entretanto o tempo necessário para fazer uma economia de aproximadamente $R \$ 20.000,00$.

Tabela 1: Dados envolvendo Financiamento

\begin{tabular}{|c|c|c|}
\hline Opção escolhida & Quantidade de respostas & Porcentagem \\
\hline Alternativa a & 9 & $(14,52 \%)$ \\
\hline Alternativa b & 4 & $(6,45 \%)$ \\
\hline Alternativa c & 2 & $(1,61 \%)$ \\
\hline Alternativa d & 5 & $(8,06 \%)$ \\
\hline Alternativa e & 42 & $(67,74 \%)$ \\
\hline
\end{tabular}

Fonte: 2021 , os autores.

Diante da análise das respostas destacadas na Tabela 1, relacionadas à escolha das alternativas, foi possível perceber uma prevalência por pagar um valor de entrada e com o saldo tendo o menor prazo, ainda que o valor da prestação fosse maior.

A partir dessas respostas, trabalhamos em sala de aula com conteúdo como progressões e funções, com o objetivo específico de modelar a situação apresentada e podermos chegar ao cálculo dos tipos e das taxas de juros cobradas em cada caso, especialmente relacionando as progressões aritméticas e geométricas aos juros simples e compostos. No trabalho em sala, foram feitas várias simulações com as situações apresentadas e foram debatidos aspectos como as vantagens e desvantagens de cada alternativa, sempre a partir da modelagem das opções. Assim, conseguimos utilizar a 
modelagem matemática como uma estratégia "para obtermos alguma compreensão acerca de determinadas situações reais" (BASSANEZI, 2012, p. 10), para então "procurarmos uma formalização que explique as relações que envolvem essas variáveis" (idem, p. 11).

Essa etapa, que durou cerca de um mês, foi caracterizada ao final pela compreensão dos estudantes a respeito da possibilidade de obter mais dados antes de fazer suas escolhas, a partir de afirmações feitas ao pesquisador. Como a alternativa "esperar e comprar à vista" havia sido a mais escolhida pelos estudantes, um dos elementos colocados em evidência nessa fase foi o tempo necessário para a economia do valor requerido, levando-se em conta um pecúlio do mesmo valor que as prestações dos itens a, b e c (já que a alternativa d não apresentava o valor da entrada), depositados em uma caderneta de poupança (OZAWA, 2013). Em todos os casos, ficou evidente que o tempo necessário seria menor que o período do financiamento, com a ressalva de que a retirada do carro só aconteceria após esse intervalo. Consideramos importante como resultado o fato de termos logrado avanços em relação à postura dos participantes, já que um dos objetivos da Educação Financeira é alcançar "compreensão, capacidade, comportamentos, atitudes e valores que permitam aos alunos tomar decisões financeiras seguras e efetivas" (MAUÉS, 2011, p. 03)

Entre os aspectos relevantes que aparecem em nossa análise dos dados, talvez o primeiro deles seja em relação ao processo de tomada de decisão dos participantes, que, em sua maioria, haviam optado por não fazer nenhum tipo de financiamento, admitindo alterar seu procedimento após a intervenção em sala. Em relação aos alunos que escolheram alguma alternativa que envolvesse empréstimo, os mesmos levaram em conta prioritariamente o prazo para o pagamento da dívida, sem considerar outros itens, como o valor da prestação ou com outros aspectos prejudiciais que pudessem surgir, como a depreciação do bem, ou as despesas com a manutenção do carro, por exemplo.

A análise nos permitiu verificar também que, apesar dos estudantes compreenderem o conceito de juros, não os relacionavam a funções e a progressões e, especialmente, não percebiam que o valor do juro aplicado se desenvolvia de forma exponencial, revelando um desconhecimento em relação aos tipos de juros existentes. Depois da intervenção realizada na sala de aula, itens importantes foram registrados pelos alunos, sendo referidos por estes como elementos que passaram a ter importância em sua decisão, como por exemplo, o tipo 
de juros, o valor das taxas cobradas e os benefícios e as perdas em relação ao tempo de retirada do carro, por exemplo.

\section{Considerações finais}

Ao iniciar este trabalho com estudantes do segundo ano do Ensino Médio, pudemos perceber a necessidade da contextualização e, consequentemente, da modelagem em um ensino de matemática voltado para a cidadania. A análise das respostas dos estudantes apontou alguns elementos que consideramos importantes tais como os aspectos aos quais já nos referimos, como o processo de tomada de decisão, a falta de relação entre juros e funções, por parte dos estudantes.

Consideramos, entretanto, que o resultado mais importante obtido com a realização deste trabalho foi exatamente a mudança entre os estudantes a respeito da necessidade de uma análise mais minuciosa antes de uma tomada de decisão como a da compra de um carro.

Por fim, gostaríamos de enfatizar nossa defesa da utilização da Educação Financeira no processo de ensino de matemática, compreendendo a mesma como a necessária intersecção entre dois elementos essenciais, a matemática financeira e o processo de tomada de decisões, configurando a promoção de elementos como a autonomia, a criticidade e, especialmente, a cidadania em nossos estudantes.

\section{Referências}

ATTIE, J. P. Relações de Poder no processo de ensino e aprendizagem. $162 \mathrm{f}$. Tese (Doutorado em Educação). Faculdade de Educação, Universidade de São Paulo. São Paulo, 2013.

BARBOSA, J.C. A contextualização e a Modelagem na educação matemática do ensino médio. In: Encontro Nacional de Educação Matemática, 8, 2004, Recife. Anais... Recife: SBEM, 2004.

BARDIN, L. Análise de conteúdo. Lisboa: Edições 70, 1977.

BASSANEZI, R. C. Temas \& Modelos. Campinas: Editora da Unicamp, 2012.

BRASIL. Lei 9394/1996. Diretrizes e Bases da Educação Nacional, de 20 de dezembro de 1996. Brasília, MEC, 1996. 
BRASIL. Ministério da Educação e do Desporto. Parâmetros Curriculares Nacionais: Matemática. Brasília, MEC/SEF,1997.

BRASIL. Ministério da Educação. Secretaria de Ensino Fundamental. Parâmetros Curriculares Nacionais do Ensino Fundamental: Introdução aos PCN. Brasília MEC/SEF, 1998.

BRASIL. Ministério da Educação. Secretaria de Educação Média e Tecnológica.

Parâmetros Curriculares Nacionais para o Ensino Médio. Brasília, MEC/SEMTEC, 1999.

BRASIL. Ministério da Educação. Base Nacional Comum Curricular (BNCC). Brasílias, MEC, 2017.

D'AMBROSIO, U. Educação para uma sociedade em transição. Campinas: Papirus, 2001.

GIL, A. C. Como elaborar projetos de pesquisa. São Paulo: Atlas, 2002.

KISTEMANN Jr., M. A. Sobre a produção de significados e a tomada de decisão de indivíduos-consumidores. 540 f. Tese (Doutorado em Educação Matemática). Instituto de Geociências e Ciências Exatas, Universidade Estadual Paulista, Rio Claro, 2011. Disponível em:

https://repositorio.unesp.br/bitstream/handle/11449/102096/kistemannjunior_ma_dr_rcla.p df?sequence=1\&isAllowed=y Acesso: 15 ago. 2012.

MACHADO, N. J. Matemática e Língua Materna: análise de uma impregnação mútua. São Paulo: Cortez, 1990.

MAUÉS, O. C. A política da OCDE para a educação e a formação docente: a nova regulação? In: Porto Alegre, v. 34, n. 1, p. 75-85, jan./abr. 2011.

NEGRI, A. L. L. Educação Financeira para o ensino médio da rede pública: uma proposta inovadora. 73 f. Dissertação (Mestrado em Educação). Centro Universitário Salesiano de São Paulo. São Paulo, 2010. Disponível em: https://unisal.br/wpcontent/uploads/2013/04/Dissertação_Ana-Lucia-Lemes-Negri.pdf Acesso: 18 ago. 2012.

OECD. Organisation for Economic Co-Operation and Development. Improving Financial Literacy: Analysis of issues and policies. Paris, 2005.

OLIVEIRA, A. A. Educação Financeira nos anos iniciais do Ensino Fundamental: como tem ocorrido na sala de aula? Dissertação (Mestrado em Educação Matemática e Tecnológica). Centro de Educação, Universidade Federal de Pernambuco, Recife, 2017. Disponível em:

https://repositorio.ufpe.br/bitstream/123456789/32214/1/DISSERTA\%c3\%87\%c3\%830\%2 0Anaelize\%20dos\%20Anjos\%20Oliveira.pdf Acesso: 16 ago. 2021. 
ONU. Programa das Nações Unidas para o Desenvolvimento. PNUD. Human development report 2019. Nova York: ONU, 2019.

ONU. A ONU e o meio ambiente. Nova York: ONU, 2020. Disponível em: https://brasil.un.org/pt-br/91223-onu-e-o-meio-ambiente Acesso 12 set. 2020.

OZAWA, N. S. Modelagem Matemática. Como vou comprar meu primeiro carro. $53 \mathrm{f}$. Dissertação (Mestrado em Matemática). PROFMAT. Universidade Federal do ABC. Santo André, 2013.

SÃO PAULO (Estado). Secretaria da Educação. Proposta curricular do Estado de São Paulo para o ensino de matemática para o ensino fundamental Ciclo II e Ensino Médio. São Paulo: SE/SP, 2008.

SBM. PROFMAT: uma reflexão e alguns resultados. Rio de Janeiro: SBM (Sociedade Brasileira de Matemática), 2017.

SKOVSMOSE, Ole. Desafios da Reflexão em Educação Matemática Crítica.

Campinas, SP: Papirus, 2000.

TEIXEIRA, J. Um estudo diagnóstico sobre a percepção da relação entre educação financeira e matemática financeira. 160 f. Tese (Doutorado em Educação Matemática). Pontifícia Universidade Católica-SP, 2015. Disponível em: http://www.sapientia.pucsp.br/arquivo Acesso: 12 abr. 2021.

\section{NOTAS}

\section{IDENTIFICAÇÃO DO TEXTO}

O presente texto é baseado no trabalho "Modelagem Matemática: como vou comprar meu primeiro carro", Dissertação de Mestrado Profissional em Matemática em Rede Nacional (Profmat) de Nilson Setsuo Ozawa, apresentado na Universidade Federal do ABC (UFABC), em 30 de setembro de 2013, elaborada sob orientação do Professor Dr. Igor Leite Freire.

\section{IDENTIFICAÇÃO DE AUTORIA}

João Paulo Attie. Doutor em Educação pela Universidade de São Paulo (2013). Professor Associado do Departamento de Matemática e Membro do Programa de Pós-Graduação em Ensino de Ciências e Matemática da Universidade Federal de Sergipe. São Cristóvão, SE, Brasil.

E-mail: jpattie@mat.ufs.br

(D) https://orcid.org/0000-0001-8411-4168

Nilson Setsuo Ozawa. Mestre em Matemática pela Universidade Federal do ABC (2013). Professor da Rede Pública Estadual de São Paulo. Mogi das Cruzes, SP, Brasil.

E-mail: niljap@yahoo.com.br

(iD) https://orcid.org/0000-0002-8343-1775

Nadir Santos Freitas. Mestranda do Programa de Pós-Graduação em Ensino de Ciências e Matemática da Universidade Federal de Sergipe. Professora da Rede Pública Estadual de Alagoas. São Cristóvão, SE, Brasil. E-mail: nadir.matematica@hotmail.com

(iD) https://orcid.org/0000-0001-9505-3356 


\section{AGRADECIMENTOS}

Não se aplica.

\section{FINANCIAMENTO}

Não se aplica.

\section{CONSENTIMENTO DE USO DE IMAGEM}

Não se aplica.

\section{APROVAÇÃO DE COMITÊ DE ÉTICA EM PESQUISA}

Não se aplica.

\section{LICENÇA DE USO}

Autores mantêm os direitos autorais e concedem à revista ENSIN@ UFMS - ISSN 2525-7056 o direito de primeira publicação, com o trabalho simultaneamente licenciado sob a Licença Creative Commons Attribution (CC BY-NC-SA 4.0), que permite compartilhar e adaptar o trabalho, para fins não comerciais, reconhecendo a autoria do texto e publicação inicial neste periódico desde que adotem a mesma licença, compartilhar igual.

\section{EDITORES}

Patricia Mirandola Garcia, Eugenia Brunilda Opazo Uribe, Gerson dos Santos Farias.

\section{HISTÓRICO}

Recebido em: 29/08/2021 - Aprovado em: 15/11/2021 - Publicado em: 15/12/2021.

\section{COMO CITAR}

ATTIE, J. P; OZAWA, N. S; FREITAS, N. S. Educação Financeira a Serviço da Cidadania. Revista ENSIN@ UFMS, Três Lagoas, v. 2, número especial, p. 90-103. 2021. 